UCLA $/ 96 /$ TEP $/ 30$

hep-th/9609132

\title{
Note on the Off-Shell Equivalence between the Linear and Non-Linear Sigma Models*
}

\author{
Hidenori SONODA ${ }^{\dagger}$ \\ Department of Physics and Astronomy, UCLA, Los Angeles, CA 90095-1547, USA
}

\begin{abstract}
As is well known, one can arrange the parameters of the $\mathrm{O}(\mathrm{N})$ non-linear sigma model to reproduce the low energy S-matrix elements of the renormalizable $\mathrm{O}(\mathrm{N})$ linear sigma model. In this note we provide details which are necessary in order to obtain the off-shell equivalence between the two theories.
\end{abstract}

September 1996

* This work was supported in part by the U.S. Department of Energy, under Contract DEAT03-88ER 40384 Mod A006 Task C.

$\dagger$ E-mail: sonoda@physics.ucla.edu; Address after 1 January 1996: Department of Physics, Kobe University, Kobe 657, Japan. 


\section{Introduction and summary}

The idea of low energy effective theories is simple: phenomena at energy less than a scale $\Lambda$ can be described by a field theory which only contains particles of mass less than $\Lambda$. The most notable example is the chiral lagrangian for the low energy pion physics [1]. The chiral lagrangian is given in terms of the pion fields such that it is invariant under the flavor $\mathrm{SU}(2) \times \mathrm{SU}(2)$. At tree level (i.e., in the soft pion limit) the lagrangian has only one parameter $f_{\pi}$, the pion decay constant, and we obtain all the consequences of current algebra simply by expanding the lagrangian and reading out the matrix elements. Away from the soft pion limit, higher order corrections have been calculated in order to achieve better fits with experiments [2]. As far as S-matrix elements are concerned, the procedure of loop expansions is well understood.

The purpose of this note is to give a clear statement of the off-shell equivalence between the $\mathrm{O}(\mathrm{N})$ linear and non-linear sigma models. The elementary fields of the linear sigma model are unconstrained scalar fields $\phi^{I}(I=1, \ldots, N)$ which transform as a vector of $\mathrm{O}(\mathrm{N})$. On the other hand the elementary fields of the non-linear sigma model are the vector $\Phi^{I}$ which are constrained by the non-linear relation

$$
\sum_{I=1}^{N} \Phi^{I} \Phi^{I}=1
$$

The off-shell equivalence means that at energies lower than the symmetry breaking scale, the correlation functions of $\phi$ 's in the linear sigma model can be reproduced by certain correlation functions in the non-linear sigma model.

Let us briefly summarize the main points of this note. First we give two points on the renormalization properties of the non-linear sigma model:

(A) The field $\Phi^{I}$ which transforms linearly under $\mathrm{O}(\mathrm{N})$ mixes with higher dimensional fields which also transform as vectors of $\mathrm{O}(\mathrm{N})$.

(B) In considering the Fourier transforms of the correlation functions, the field $\Phi^{I}$ is not renormalized linearly: we must introduce counterterms to remove the ultraviolet (UV) divergences due to two or more $\Phi$ 's at the same point in space.

The first point is nothing new. Since the non-linear sigma model is non-renormalizable, the field $\Phi^{I}$ cannot be renormalized multiplicatively, but it mixes with an infinite number of higher dimensional fields which transform as vectors under $\mathrm{O}(\mathrm{N})$. The second point, which is familiar from the renormalization of composite fields in renormalizable field theories 
(see, for example, ref. [3]), has not been emphasized before in the literature. This point is not only important for the removal of UV divergences but also for obtaining the off-shell equivalence. See $\left(\mathrm{B}^{\prime}\right)$ below.

Similarly, we give two points on the off-shell equivalence:

$\left(\mathrm{A}^{\prime}\right)$ The elementary field $\phi^{I}$ of the linear sigma model corresponds to a linear combination of the fields in the non-linear sigma model all of which transform as vectors of $\mathrm{O}(\mathrm{N})$. More specifically, $\phi^{I}$ corresponds to a linear combination of the non-linear field $\Phi^{I}$ and fields with two or more derivatives.

$\left(\mathrm{B}^{\prime}\right)$ For the off-shell equivalence, we must also introduce finite counterterms corresponding to two or more fields at the same point in space.

The loop expansion of the $\mathrm{O}(\mathrm{N})$ non-linear sigma model was discussed long ago by Appelquist and Bernard [4] and by Akhoury and Yao [5]. The results of this note are refinements of some of the results of the above mentioned works.

The present paper is organized as follows. In sect. 2, we summarize the one-loop corrections to the two- and four-point correlation functions of $\Phi$ 's in the $\mathrm{O}(\mathrm{N})$ non-linear sigma model. We only give enough details to clarify our points (A,B) above. In sect. 3, we consider the one-loop corrections to the two- and four-point correlation functions of $\phi$ 's in the $\mathrm{O}(\mathrm{N})$ linear sigma model, and take their low energy approximations. In sect. 4 we compare the results of sects. 2 and 3, and determine the parameters of the low energy effective theory to obtain the off-shell equivalence. In sect. 5 we give concluding remarks.

Throughout this note we will use the dimension regularization with

$$
D \equiv 4-\epsilon
$$

For simplicity, we will use the euclidean metric.

\section{The loop expansion of the $\mathrm{O}(\mathrm{N})$ non-linear sigma model}

The $\mathrm{O}(\mathrm{N})$ non-linear sigma model is defined by the lagrangian

$$
\begin{aligned}
\mathcal{L}_{e f f}= & \frac{1}{2 g_{-2}} \partial_{\mu} \Phi^{I} \partial_{\mu} \Phi^{I} \\
+\frac{1}{g_{-2}^{2}}( & G_{-4}^{(1)}\left(\partial_{\mu} \Phi^{I} \partial_{\mu} \Phi^{I}\right)^{2}+G_{-4}^{(2)} \partial_{\mu} \Phi^{I} \partial_{\nu} \Phi^{I} \partial_{\mu} \Phi^{J} \partial_{\nu} \Phi^{J} \\
& \left.\quad+G_{-4}^{(3)} \partial^{2} \Phi^{I} \partial^{2} \Phi^{I}\right)+\ldots,
\end{aligned}
$$


where the field $\Phi^{I}(I=1, \ldots, N)$ takes values on the unit sphere $S^{N}$, and the summation over the repeated vector indices $I, J$ is implied. The dots represent terms with six or more derivatives. There are only three terms possible with four derivatives. The three parameters are given as

$$
G_{-4}^{(i)}=\frac{z^{(i)}}{\epsilon} g_{-2}^{2}+g_{-4}^{(i)}
$$

where $z^{(i)}, g_{-4}^{(i)}$ are finite constants. The constants $z^{(i)}$ are chosen to remove the oneloop UV divergences. The finite parameter $g_{-4}^{(i)}$ satisfies the renormalization group (RG) equation:

$$
\frac{d}{d t} g_{-4}^{(i)}=(-4+\epsilon) g_{-4}^{(i)}-z^{(i)} g_{-2}^{2}
$$

Before discussing the the renormalization of the field $\Phi^{I}$, we must make an important remark. The fields $\Phi^{I}(I=1, \ldots, N)$ satisfy the non-linear constraint (1.1). Given the convention

$$
\left\langle\Phi^{i}\right\rangle=0 \quad(i=1, \ldots, N-1)
$$

a standard parametrization is

$$
\Phi^{i}=\sqrt{g_{-2}} \pi^{i}(i=1, \ldots, N-1), \quad \Phi^{N}=\sqrt{1-g_{-2} \vec{\pi} \cdot \vec{\pi}}
$$

where $\pi^{i}$ are $\mathrm{N}-1$ independent fields. But what parametrization we use is irrelevant as long as we use the dimension regularization, since the jacobian of the infinitesimal field transformation

$$
\begin{aligned}
\pi^{i} \rightarrow(1+ & \left.\epsilon_{0}\left(\pi^{2}\right)\right) \pi^{i}+\epsilon_{1}^{i j k}\left(\pi^{2}\right) \partial_{\mu} \pi^{j} \partial_{\mu} \pi^{k}+\epsilon_{2}^{i j}\left(\pi^{2}\right) \partial^{2} \pi^{j} \\
& + \text { (higher order derivatives) }
\end{aligned}
$$

is unity. (See ref. [6] for a recent discussion.) Therefore, we need not discuss the fields $\pi^{i}$ which give a non-linear realization of $\mathrm{O}(\mathrm{N})$, but we only need to discuss linear representations of $\mathrm{O}(\mathrm{N})$ such as $\Phi^{I}$ and their derivatives.

The renormalization of $\Phi^{I}$ must be done in two steps. First, we must renormalize the field linearly as

$$
\left[\Phi^{I}\right]=\Phi^{I}+\frac{g_{-2}}{\epsilon}\left\{a_{1}\left(\partial_{\mu} \Phi^{J} \partial_{\mu} \Phi^{J}\right) \Phi^{I}+a_{2} \partial^{2} \Phi^{I}\right\}+\ldots
$$

where $a_{1}, a_{2}$ are finite constants, and we have suppressed the terms with more than two derivatives. The above linear renormalization does not remove all the UV divergences 
of the Fourier transforms of the correlation functions. We must also subtract the UV singularities corresponding to two or more fields at the same point in space. At one-loop level we only need counterterms for the product of two $\Phi$ 's 1 :

$$
\begin{aligned}
& \left\langle\left[\Phi^{I}\left(p_{1}\right) \Phi^{J}\left(p_{2}\right)\right]\right\rangle=\left\langle\left[\Phi^{I}\right]\left(p_{1}\right)\left[\Phi^{J}\right]\left(p_{2}\right)\right\rangle \\
& \quad+g_{-2}^{2} \frac{1}{\epsilon}\left(b_{1} \delta^{I J}+b_{2}\left\langle\Phi^{I} \Phi^{J}-\frac{\delta^{I J}}{N}\right\rangle\right)(2 \pi)^{D} \delta^{(D)}\left(p_{1}+p_{2}\right) \\
& \left\langle\left[\Phi^{I}\left(p_{1}\right) \Phi^{J}\left(p_{2}\right) \Phi^{K}\left(p_{3}\right) \Phi^{L}\left(p_{4}\right)\right]\right\rangle^{c} \\
& =\left\langle\left[\Phi^{I}\right]\left(p_{1}\right)\left[\Phi^{J}\right]\left(p_{2}\right)\left[\Phi^{K}\right]\left(p_{3}\right)\left[\Phi^{L}\right]\left(p_{4}\right)\right\rangle^{c} \\
& \quad+g_{-2}^{2} \frac{b_{2}}{\epsilon}\left(\left\langle\left(\Phi^{I} \Phi^{J}\right)\left(p_{1}+p_{2}\right) \Phi^{K}\left(p_{3}\right) \Phi^{L}\left(p_{4}\right)\right\rangle^{c}+(5 \text { other terms })\right),
\end{aligned}
$$

where the correlation functions are given in the momentum space. The correlation functions of more number of $\Phi$ 's are renormalized analogously. The finite constants $b_{1,2}$ are determined to remove the remaining one-loop divergences. Note that in the coordinate space these counterterms would be proportional to delta functions.

We have introduced the seven renormalization constants $z^{(1,2,3)}, a_{1,2}, b_{1,2}$ to remove the one-loop UV divergences. (See Appendix A for an explicit expression of the oneloop UV divergences.) We can observe that not all the renormalization constants are independent. This is a consequence of equations of motion. Equations of motion have been discussed extensively in the literature, and we give a short summary in Appendix B. For our purposes we need the following two equations of motion, derived with only the leading two-derivative term in the lagrangian (2.1).

The first equation of motion

$$
\begin{aligned}
& \left\langle\left\{\left(\partial \Phi^{I} \partial \Phi^{I}\right) \Phi^{I}+\partial^{2} \Phi^{I}\right\}(x) \Phi^{I_{1}}\left(x_{1}\right) \ldots \Phi^{I_{n}}\left(x_{n}\right)\right\rangle \\
& \quad=\sum_{k=1}^{n} \delta^{(D)}\left(x-x_{k}\right)\left\langle\Phi^{I_{1}}\left(x_{1}\right) \ldots g_{-2}\left(-\delta^{I I_{k}}+\Phi^{I} \Phi^{I_{k}}\right)\left(x_{k}\right) \ldots \Phi^{I_{n}}\left(x_{n}\right)\right\rangle
\end{aligned}
$$

implies that the correlation functions of $\Phi$ 's are invariant under the shift

$$
\Delta a_{1}=\Delta a_{2}=\delta, \quad \Delta b_{1}=2(1-1 / N) \delta, \quad \Delta b_{2}=-2 \delta .
$$

1 At higher orders of the loop expansion we need counterterms for the products of more than two fields. 
Similarly, the second equation of motion

$$
\begin{aligned}
& -\int d^{D} x\left\langle\left\{-\left(\partial \Phi^{I} \partial \Phi^{I}\right)^{2}+\partial^{2} \Phi^{I} \partial^{2} \Phi^{I}\right\}(x) \Phi^{I_{1}}\left(x_{1}\right) \ldots \Phi^{I_{n}}\left(x_{n}\right)\right\rangle \\
& =\sum_{k=1}^{n}\left\langle\Phi^{I_{1}}\left(x_{1}\right) \ldots g_{-2}\left\{\left(\partial \Phi^{I} \partial \Phi^{I}\right) \Phi^{I_{k}}+\partial^{2} \Phi^{I_{k}}\right\}\left(x_{k}\right) \ldots \Phi^{I_{n}}\left(x_{n}\right)\right\rangle
\end{aligned}
$$

implies the invariance of the correlation functions under

$$
\Delta z^{(1)}=-\Delta z^{(3)}=\Delta a_{1}=\Delta a_{2} .
$$

To remove the ambiguities (2.10), (2.12), it is convenient to adopt a convention

$$
z^{(3)}=b_{2}=0 .
$$

Under the above convention (2.13), we can determine the renormalization constants uniquely as

$$
\begin{aligned}
& z^{(1)}=\frac{1}{(4 \pi)^{2}}\left(\frac{1}{3}+\frac{N-4}{2}\right), \quad z^{(2)}=\frac{1}{(4 \pi)^{2}} \frac{2}{3}, \\
& a_{1}=\frac{1}{(4 \pi)^{2}}\left(\frac{1}{2}+\frac{N-4}{2}\right), \quad a_{2}=\frac{1}{(4 \pi)^{2}}\left(-\frac{3}{2}-\frac{N-4}{2}\right), \\
& b_{1}=2 a_{2} .
\end{aligned}
$$

The two- and four-point functions of $\Phi^{i}(i=1, \ldots, N-1)$ are now given by

$$
\begin{aligned}
& \frac{1}{g_{-2}}\left\langle\left[\Phi^{i}\left(p_{1}\right) \Phi^{j}\left(p_{2}\right)\right]\right\rangle \simeq \delta^{i j} \frac{1}{p_{1}^{2}}\left(1-\frac{2 g_{-4}^{(3)}}{g_{-2}} p_{1}^{2}\right), \\
& \frac{1}{g_{-2}^{2}}\left\langle\left[\Phi^{i}\left(p_{1}\right) \Phi^{j}\left(p_{2}\right) \Phi^{k}\left(p_{3}\right) \Phi^{l}\left(p_{4}\right)\right]\right\rangle^{c} \\
& \simeq \frac{1}{g_{-2}^{2}}\left\langle\left[\Phi^{i}\left(p_{1}\right) \Phi^{j}\left(p_{2}\right) \Phi^{k}\left(p_{3}\right) \Phi^{l}\left(p_{4}\right)\right]\right\rangle_{1-\text { loop MS }}^{c} \\
& +\frac{1}{p_{1}^{2} p_{2}^{2} p_{3}^{2} p_{4}^{2}}\left[\delta ^ { i j } \delta ^ { k l } \left\{-8\left(g_{-4}^{(1)}+g_{-4}^{(3)}\right)\left(p_{1} p_{2}\right)\left(p_{3} p_{4}\right)\right.\right. \\
& \quad-4 g_{-4}^{(2)}\left(\left(p_{1} p_{3}\right)\left(p_{2} p_{4}\right)+\left(p_{1} p_{4}\right)\left(p_{2} p_{3}\right)\right) \\
& \left.\left.\quad+2 g_{-4}^{(3)}\left(p_{1}^{2}+p_{2}^{2}\right)\left(p_{3}^{2}+p_{4}^{2}\right)\right\}+(\mathrm{t}, \mathrm{u}-\text { channels })\right],
\end{aligned}
$$

where we have suppressed the factor $(2 \pi)^{4} \delta^{(4)}\left(p_{1}+\ldots\right)$ of momentum conservation. The first term on the right-hand side of eqn. (2.15B) stands for the correlation function up to 
one-loop in the minimal subtraction (MS) scheme in which we take $g_{-4}^{(i)}=0$. For a later convenience we introduce a notation $\Gamma_{s}^{N L}$ by

$$
\begin{aligned}
& \frac{1}{g_{-2}^{2}}\left\langle\left[\Phi^{i}\left(p_{1}\right) \Phi^{j}\left(p_{2}\right) \Phi^{k}\left(p_{3}\right) \Phi^{l}\left(p_{4}\right)\right]\right\rangle_{1 \text {-loop MS }}^{c} \\
& =\frac{1}{p_{1}^{2} p_{2}^{2} p_{3}^{2} p_{4}^{2}}\left[\delta^{i j} \delta^{k l}\left(-g_{-2} s-\Gamma_{s}^{N L}\left(g_{-2} ; p_{1}, \ldots, p_{4}\right)\right)+(\mathrm{t}, \mathrm{u} \text {-channels })\right] .
\end{aligned}
$$

We note that the four-point function is free of UV divergences without the $b_{1}$ counterterm, but the two-point function would have a UV divergence proportional to a delta function in space. The necessity of the $b_{1}$ counterterm has been missed in the previous literature.

Before concluding this section, we note the inhomogeneous RG equation satisfied by the correlation functions:

$$
\begin{gathered}
\left(\frac{d}{d t}+8\right)\left\langle\left[\Phi^{I}\left(p_{1}\right) \Phi^{J}\left(p_{2}\right)\right]\right\rangle=g_{-2}\left(\left\langle\left\{a_{1}(\partial \Phi \partial \Phi) \Phi^{I}+a_{2} \partial^{2} \Phi^{I}\right\} \Phi^{J}\right\rangle+(I \leftrightarrow J)\right) \\
\quad+b_{1} g_{-2}^{2} \delta^{I J}(2 \pi)^{4} \delta^{(4)}\left(p_{1}+p_{2}\right), \\
\left(\frac{d}{d t}+16\right)\left\langle\left[\Phi^{I}\left(p_{1}\right) \Phi^{J}\left(p_{2}\right) \Phi^{K}\left(p_{3}\right) \Phi^{L}\left(p_{4}\right)\right]\right\rangle^{c} \\
=g_{-2}\left(\left\langle\left\{a_{1}(\partial \Phi \partial \Phi) \Phi^{I}+a_{2} \partial^{2} \Phi^{I}\right\} \Phi^{J} \Phi^{K} \Phi^{L}\right\rangle^{c}+(3 \text { other terms })\right),
\end{gathered}
$$

where $a_{1,2}, b_{1}$ are given by (2.14).

\section{The low energy expansion of the linear sigma model}

The $\mathrm{O}(\mathrm{N})$ linear sigma model is defined by the lagrangian

$$
\mathcal{L}=\frac{1}{2} Z \partial_{\mu} \phi^{I} \partial_{\mu} \phi^{I}+\frac{Z_{\lambda} \lambda}{8}\left(Z \phi^{I} \phi^{I}-\frac{Z_{m}}{Z_{\lambda}} v^{2}\right)^{2}
$$

where the elementary fields $\phi^{I}(I=1, \ldots, N)$ are unconstrained, and we have written the renormalization constants $Z, Z_{\lambda}, Z_{m}$ explicitly. A mass squared parameter is defined by

$$
m^{2} \equiv \lambda v^{2}
$$

It is convenient to adopt the MS scheme to determine the renormalization constants. At one-loop we find

$$
Z \simeq 1, \quad Z_{\lambda} \simeq 1+\frac{\lambda}{(4 \pi)^{2} \epsilon}(N+8), \quad Z_{m} \simeq 1+\frac{\lambda}{(4 \pi)^{2} \epsilon}(N+2) .
$$


These imply the following one-loop RG equations:

$$
\frac{d}{d t} \phi^{I}=\phi^{I}, \quad \frac{d}{d t} \lambda=-\frac{N+8}{(4 \pi)^{2}} \lambda^{2}, \quad \frac{d}{d t} m^{2}=\left(2-\frac{N+2}{(4 \pi)^{2}} \lambda\right) m^{2} .
$$

For a later convenience, we note that

$$
\frac{d}{d t} \frac{\lambda}{m^{2}}=\left(-2-\frac{6}{(4 \pi)^{2}} \lambda\right) \frac{\lambda}{m^{2}}
$$

We choose the vacuum such that

$$
\left\langle\phi^{i}\right\rangle=0 \quad(i=1, \ldots, N-1)
$$

corresponding to the convention (2.4).

Our goal is to obtain the two- and four-point functions of the elementary fields $\phi^{i}(i=$ $1, \ldots, N-1)$ up to one-loop. Especially we wish to obtain their low energy approximations so that we can compare them with the correlation functions obtained in the previous section.

At tree-level the four-point vertex (in an obvious notation) is given by

$$
\begin{aligned}
-\Gamma_{\text {tree }}^{(4)}\left(p_{1}, \ldots, p_{4}\right) & =\lambda\left[\delta^{i j} \delta^{k l}\left(\frac{m^{2}}{s+m^{2}}-1\right)+(\mathrm{t}, \mathrm{u} \text {-channels })\right] \\
& \simeq \lambda\left[\delta^{i j} \delta^{k l}\left(-\frac{s}{m^{2}}+\left(\frac{s}{m^{2}}\right)^{2}\right)+(\mathrm{t}, \mathrm{u} \text {-channels })\right],
\end{aligned}
$$

where $s \equiv\left(p_{1}+p_{2}\right)^{2}$, and we have approximated the vertex up to four derivatives. The above gives the relation

$$
g_{-2} \simeq \frac{\lambda}{m^{2}}=\frac{1}{v^{2}}
$$

at lowest order in $\lambda$.

The one-loop calculations are straightforward and can be found elsewhere (for example, [4]). Up to one-loop the two-point function of $\phi^{i}(i=1, \ldots, N-1)$ is given by

$$
\left\langle\phi^{i}\left(p_{1}\right) \phi^{j}\left(p_{2}\right)\right\rangle=\frac{\delta^{i j}}{p_{1}^{2}}\left(1-\frac{\lambda}{(4 \pi)^{2}} \frac{1}{2}+\frac{\lambda}{m^{2}} \frac{1}{(4 \pi)^{2}} \frac{1}{6} p_{1}^{2}+\mathrm{O}\left(\left(p_{1}^{2}\right)^{2}\right)\right),
$$

where as usual we have suppressed the factor $(2 \pi)^{4} \delta^{(4)}\left(p_{1}+p_{2}\right)$ of momentum conservation.

The one-loop calculation of the four-point function of $\phi^{i}(i=1, \ldots, N-1)$ is more elaborate. But the calculation can be simplified by comparing the one-loop corrections in the linear sigma model with those in the non-linear sigma model. As an example, 
let us consider the one-loop contribution to the vertex from the Feynman diagrams in Fig. 1. (The solid line corresponds to the field $\phi^{i}(i=1, \ldots, N-1)$, and the broken line corresponds to the field $\sigma \equiv \phi^{N}-v$.)

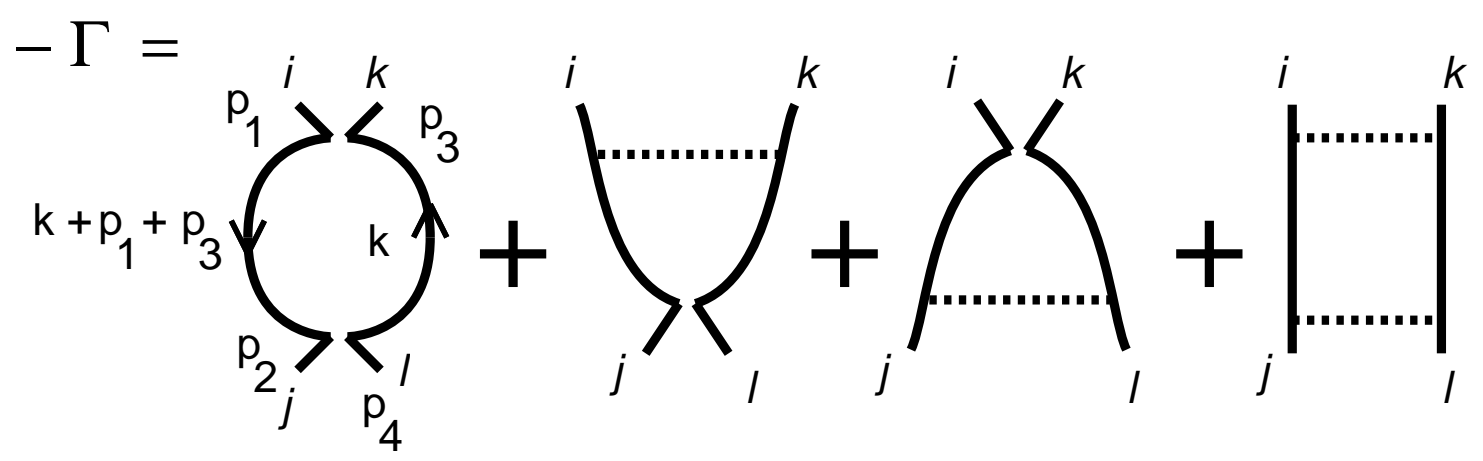

Fig. 1

We easily find

$$
\begin{aligned}
-\Gamma & =\delta^{i j} \delta^{k l} \lambda^{2} \int_{k} \frac{1}{k^{2}\left(k+p_{1}+p_{3}\right)^{2}}\left[1-\frac{m^{2}}{m^{2}+\left(k+p_{3}\right)^{2}}\right. \\
& \left.-\frac{m^{2}}{m^{2}+\left(k-p_{4}\right)^{2}}+\frac{m^{2}}{m^{2}+\left(k+p_{3}\right)^{2}} \cdot \frac{m^{2}}{m^{2}+\left(k-p_{4}\right)^{2}}\right] \\
= & \delta^{i j} \delta^{k l}\left(G+\frac{\lambda^{2}}{m^{4}} I\right),
\end{aligned}
$$

where

$$
G \equiv \frac{\lambda^{2}}{m^{4}} \int_{k} \frac{\left(k+p_{3}\right)^{2}\left(k-p_{4}\right)^{2}}{k^{2}\left(k+p_{1}+p_{3}\right)^{2}}
$$

corresponds to the contribution of the one-loop graph (Fig. 2) in the low energy effective theory with $g_{-2}=\frac{\lambda}{m^{2}}$, and

$$
I \equiv \int_{k} \frac{\left(k+p_{3}\right)^{2}\left(k-p_{4}\right)^{2}}{k^{2}\left(k+p_{1}+p_{3}\right)^{2}}\left[\frac{m^{2}}{m^{2}+\left(k+p_{3}\right)^{2}} \frac{m^{2}}{m^{2}+\left(k-p_{4}\right)^{2}}-1\right] .
$$

As far as the contributions up to fourth order in external momenta are concerned, all the non-local dependence is contained in $G$, and the integral $I$ is local, i.e., $I$ is a polynomial of the external momenta. By expanding the integrand of $I$ in external momenta, we can obtain a low energy approximation of $I$ without encountering infrared divergences.2

2 The same technique was used in a proof of the decoupling of massive modes. See Chapter 8 of Collins's textbook [3]. 


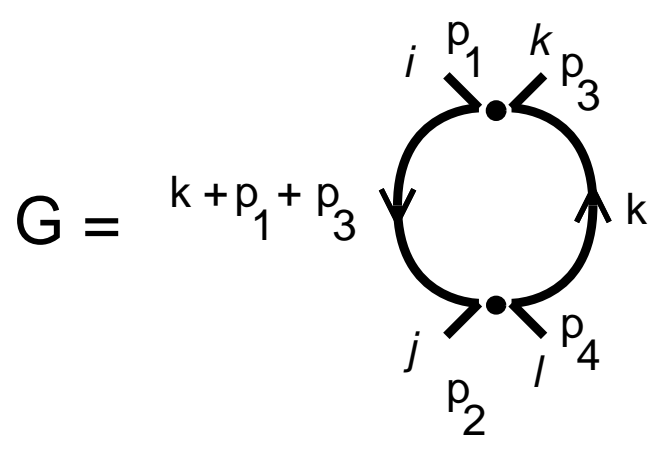

Fig. 2

The low energy approximations of the other graphs can be evaluated analogously. The final result is

$$
\begin{aligned}
& -\Gamma_{1 \text {-loop }}^{(4)}\left(p_{1}, \ldots, p_{4}\right) \simeq \delta^{i j} \delta^{k l}\left[-\Gamma_{s}^{N L}\left(\frac{\lambda}{m^{2}} ; p_{1}, \ldots, p_{4}\right)\right. \\
& +\frac{\lambda^{2}}{m^{4}} \frac{1}{(4 \pi)^{2}}\left\{-m^{2} s\left(3 \ln -\frac{5}{2}\right)+\left(\frac{4}{3} \ln -\frac{82}{9}\right)\left(p_{1} p_{2}\right)\left(p_{3} p_{4}\right)\right. \\
& \quad+\left(\frac{4}{3} \ln -\frac{34}{9}\right)\left(\left(p_{1} p_{3}\right)\left(p_{2} p_{4}\right)+\left(p_{1} p_{4}\right)\left(p_{2} p_{3}\right)\right) \\
& \quad+\left(\ln -\frac{23}{6}\right) s\left(p_{1}^{2}+\ldots+p_{4}^{2}\right) \\
& \left.\left.\quad+\left(-\frac{1}{2} \ln +\frac{17}{18}\right)\left(p_{1}^{2}+p_{2}^{2}\right)\left(p_{3}^{2}+p_{4}^{2}\right)\right\}\right]+(\mathrm{t}, \mathrm{u} \text {-channels })
\end{aligned}
$$

where $\Gamma_{s}^{N L}$ is defined in eqn. (2.16), and

$$
\ln \equiv \ln \frac{m^{2} \mathrm{e}^{\gamma}}{4 \pi} .
$$

We have only kept the terms up to fourth order in external momenta. It is trivial to construct the connected four-point correlation function $\left\langle\phi^{i}\left(p_{1}\right) \ldots \phi^{l}\left(p_{4}\right)\right\rangle^{c}$ from eqs. (3.9), (3.13).

\section{Off-shell equivalence}

We now wish to construct the correlation functions in the non-linear sigma model which reproduce the low energy approximations of the correlation functions in the linear sigma model. 
The $\mathrm{O}(\mathrm{N})$ symmetry demands that the elementary field $\phi^{I}$ of the linear sigma model corresponds to a linear combination of the fields which transform as vectors of $O(N)$ in the non-linear sigma model:

$$
\begin{array}{r}
\phi_{\text {eff }}^{I}=z\left(\lambda, m^{2}\right)\left\{\left[\Phi^{I}\right]+g_{-2} A_{1}\left(\lambda, m^{2}\right)\left(\partial_{\mu} \Phi^{J} \partial_{\mu} \Phi^{J}\right) \Phi^{I}\right. \\
\left.+g_{-2} A_{2}\left(\lambda, m^{2}\right) \partial^{2} \Phi^{I}+\ldots\right\} .
\end{array}
$$

To assure the off-shell equivalence we also need finite counterterms which correspond to products of two $\Phi$ 's at the same point in space. Thus, the off-shell equivalence between the two theories means that we can choose the nine parameters $g_{-2}, g_{-4}^{(1,2,3)}, z, A_{1,2}$, and $B_{1,2}$ such that

$$
\begin{aligned}
& \left\langle\phi^{I}\left(p_{1}\right) \phi^{J}\left(p_{2}\right)\right\rangle=\left\langle\left[\phi_{e f f}^{I}\left(p_{1}\right) \phi_{\text {eff }}^{J}\left(p_{2}\right)\right]\right\rangle+(2 \pi)^{4} \delta^{(4)}\left(p_{1}+p_{2}\right) \\
& \quad \times g_{-2}^{2}\left\{\delta^{I J} B_{1}\left(\lambda, m^{2}\right)+B_{2}\left(\lambda, m^{2}\right)\left\langle\Phi^{I} \Phi^{J}-\frac{\delta^{I J}}{N}\right\rangle\right\} \\
& \left\langle\phi^{I}\left(p_{1}\right) \phi^{J}\left(p_{2}\right) \phi^{K}\left(p_{3}\right) \phi^{L}\left(p_{4}\right)\right\rangle^{c}=\left\langle\left[\phi_{e f f}^{I}\left(p_{1}\right) \phi_{e f f}^{J}\left(p_{2}\right) \phi_{\text {eff }}^{K}\left(p_{3}\right) \phi_{e f f}^{L}\left(p_{4}\right)\right]\right\rangle^{c} \\
& \quad+g_{-2}^{2} B_{2}\left(\lambda, m^{2}\right)\left\{\left\langle\left(\Phi^{I} \Phi^{J}\right)\left(p_{1}+p_{2}\right) \Phi^{K}\left(p_{3}\right) \Phi^{L}\left(p_{4}\right)\right\rangle^{c}+(5 \text { other terms })\right\} .
\end{aligned}
$$

As in sect. 2, the equations of motion (2.9), (2.11) imply that only seven of the nine parameters are independent, and following (2.13) we adopt the convention

$$
g_{-4}^{(3)}=B_{2}=0 \text {. }
$$

From the results of the previous two sections, we obtain the following results to order $\lambda^{2}$ :

$$
\begin{aligned}
g_{-2} & \simeq \frac{\lambda}{m^{2}}\left(1+\frac{\lambda}{(4 \pi)^{2}}\left(3 \ln -\frac{7}{2}\right)\right) \\
g_{-4}^{(1)} & \simeq-\frac{1}{2} \frac{\lambda}{m^{4}}+\frac{\lambda^{2}}{m^{4}} \frac{1}{(4 \pi)^{2}}\left(-\frac{1}{6} \ln +\frac{41}{36}\right) \\
g_{-4}^{(2)} & \simeq \frac{\lambda^{2}}{m^{4}} \frac{1}{(4 \pi)^{2}}\left(-\frac{1}{3} \ln +\frac{17}{18}\right) \\
g_{-2}^{2} A_{1} & \simeq-\frac{1}{2} \frac{\lambda}{m^{4}}+\frac{\lambda^{2}}{m^{4}} \frac{1}{(4 \pi)^{2}}\left(-\frac{1}{4} \ln +\frac{17}{36}\right) \\
g_{-2}^{2} A_{2} & \simeq \frac{1}{2} \frac{\lambda}{m^{4}}+\frac{\lambda^{2}}{m^{4}} \frac{1}{(4 \pi)^{2}}\left(\frac{3}{4} \ln -\frac{127}{36}\right) \\
g_{-2}^{2} B_{1} & \simeq \frac{\lambda}{m^{4}}+\frac{\lambda^{2}}{m^{4}} \frac{1}{(4 \pi)^{2}}\left(\frac{3}{2} \ln -\frac{62}{9}\right),
\end{aligned}
$$


where $\ln$ is defined by eqn. (3.14). The normalization constant $z$ is given, to order $\lambda$, by

$$
z \simeq 1-\frac{\lambda}{(4 \pi)^{2}} \frac{1}{4}
$$

Let us note the consistency between the dependence of $\ln m^{2}$ in the above results and the one-loop RG. (The one-loop RG equations for the non-linear sigma model are given by eqs. (2.3) and (2.17). Those for the linear sigma model are given by eqs. (3.4), (3.5).) Since the anomalous dimension of the field $\phi^{I}$ vanishes to order $\lambda$, the normalization constant $z$ should be independent of the $\ln m^{2}$, which is consistent with eqn. (4.5). Because of eqn. (3.5), eqn. (4.4a) is consistent with

$$
\frac{d}{d t} g_{-2}=-2 g_{-2}
$$

Eqs. (4.4b, $)$ give

$$
\begin{aligned}
& \left(\frac{d}{d t}+4\right) g_{-4}^{(1)} \simeq-\frac{g_{-2}^{2}}{(4 \pi)^{2}}\left(\frac{1}{3}+\frac{N-4}{2}\right)=-z^{(1)} g_{-2}^{2} \\
& \left(\frac{d}{d t}+4\right) g_{-4}^{(2)} \simeq-\frac{g_{-2}^{2}}{(4 \pi)^{2}} \frac{2}{3}=-z^{(2)} g_{-2}^{2}
\end{aligned}
$$

which agree with eqn. (2.3) due to eqs. (2.14). The constants $A_{1,2}, B_{1}$ satisfy

$$
\begin{aligned}
& \left(\frac{d}{d t}+4\right) g_{-2}^{2} A_{1} \simeq-\frac{g_{-2}^{2}}{(4 \pi)^{2}}\left(\frac{1}{2}+\frac{N-4}{2}\right)=-a_{1} g_{-2}^{2} \\
& \left(\frac{d}{d t}+4\right) g_{-2}^{2} A_{2} \simeq \frac{g_{-2}^{2}}{(4 \pi)^{2}}\left(\frac{3}{2}+\frac{N-4}{2}\right)=-a_{2} g_{-2}^{2} \\
& \left(\frac{d}{d t}+4\right) g_{-2}^{2} B_{1} \simeq \frac{g_{-2}^{2}}{(4 \pi)^{2}}(3+(N-4))=-b_{1} g_{-2}^{2} .
\end{aligned}
$$

Hence, the inhomogeneous counterterms given by $A_{1,2}, B_{1}$ cancel the inhomogeneous terms in the $\mathrm{RG}$ eqs. (2.17).

\section{Concluding remarks}

In this note we have shown how to achieve the off-shell equivalence between the $\mathrm{O}(\mathrm{N})$ linear and non-linear sigma models by choosing the parameters of the non-linear sigma model as appropriate functions of $\lambda$ and $m^{2}$ of the linear sigma model. At one-loop level we have determined the seven parameters $g_{-2}, g_{-4}^{(1,2)}, z, A_{1,2}$, and $B_{1}$ in terms of $\lambda, m^{2}$ as in eqs. (4.4), (4.5). 
The emphasis of this note is that the off-shell equivalence can be achieved by introducing all possible counterterms allowed by the $\mathrm{O}(\mathrm{N})$ invariance. We especially emphasize the importance of the inhomogeneous counterterm proportional to $B_{1}$ in eqn. (4.2a). At higher loop orders we need to introduce more inhomogeneous counterterms corresponding to products of more than two fields at the same point in space. Their role will increase at higher orders.

Another point of emphasis is the irrelevance of any particular way of parametrizing the fields $\Phi^{I}$ which satisfy the non-linear constraint (1.1). We only need to be concerned with the linear representations of $\mathrm{O}(\mathrm{N})$, but not with their non-linear realizations.

Out of the seven parameters of the non-linear sigma model, only three, i.e, $g_{-2}$ and $g_{-4}^{(1,2)}$, affect the S-matrix elements. (See ref. [6] for a recent discussion.) As is well known, the S-matrix elements are independent of the choice of a particular interpolating field. Hence, the parameters $z$ and $A_{1,2}$ are irrelevant. The counterterm proportional to $B_{1}$ vanishes when the external legs are amputated, and the momenta are put on the mass shell.

Acknowledgment: The present work was done while the author was visiting the Theory Group of KEK in Japan. He would like to thank the group for a stimulating atmosphere. 


\section{Appendix A. One-loop calculations in the $\mathrm{O}(\mathrm{N})$ non-linear sigma model}

For completeness we give the results of the one-loop calculations in the $\mathrm{O}(\mathrm{N})$ nonlinear sigma model [4] [5]. The two- and four-point functions of $\Phi^{i}(i=1, \ldots, N-1)$ up to one-loop level are given as follows:

$$
\begin{aligned}
\frac{1}{g_{-2}}\langle & {\left.\left[\Phi^{i}\left(p_{1}\right) \Phi^{j}\left(p_{2}\right)\right]\right\rangle } \\
= & \frac{\delta^{i j}}{p_{1}^{2}}\left[1+\left(p_{1}^{2}\right)^{2}\left\{-2 G_{-4}^{(3)}+\frac{g_{-2}^{2}}{\epsilon}\left(-2 a_{2}+b_{1}-\frac{b_{2}}{N}\right)\right\}\right] \\
\frac{1}{g_{-2}^{2}}\langle & \left.\left\langle\Phi^{i}\left(p_{1}\right) \Phi^{j}\left(p_{2}\right) \Phi^{k}\left(p_{3}\right) \Phi^{l}\left(p_{4}\right)\right]\right\rangle^{c} \\
= & \frac{1}{g_{-2}^{2}}\left\langle\Phi^{i}\left(p_{1}\right) \Phi^{j}\left(p_{2}\right) \Phi^{k}\left(p_{3}\right) \Phi^{l}\left(p_{4}\right)\right\rangle_{1-\text { loop MS }}^{c}+\frac{1}{p_{1}^{2} p_{2}^{2} p_{3}^{2} p_{4}^{2}}\{ \\
& \delta^{i j} \delta^{k l}\left[\left\{\frac{g_{-2}^{2}}{(4 \pi)^{2} \epsilon}\left(\frac{8}{3}+4(N-4)\right)-8\left(G_{-4}^{(1)}+G_{-4}^{(3)}\right)\right\}\left(p_{1} p_{2}\right)\left(p_{3} p_{4}\right)\right. \\
& +\left(\frac{g_{-2}^{2}}{(4 \pi)^{2} \epsilon} \frac{8}{3}-4 G_{-4}^{(2)}\right)\left(\left(p_{1} p_{3}\right)\left(p_{2} p_{4}\right)+\left(p_{1} p_{4}\right)\left(p_{2} p_{3}\right)\right) \\
+ & \left\{\frac{g_{-2}^{2}}{\epsilon}\left(-\frac{1+(N-4)}{(4 \pi)^{2}}+2 a_{1}+b_{2}\right)+2 G_{-4}^{(3)}\right\}\left(p_{1}^{2}+p_{2}^{2}\right)\left(p_{3}^{2}+p_{4}^{2}\right) \\
+ & \left.\left.\frac{g_{-2}^{2}}{\epsilon}\left(\frac{2+(N-4)}{(4 \pi)^{2}}-a_{1}+a_{2}\right) s\left(p_{1}^{2}+\ldots+p_{4}^{2}\right)\right]+(\mathrm{t}, \mathrm{u} \text {-channels })\right\},
\end{aligned}
$$

where we have suppressed the factor $(2 \pi)^{D} \delta^{(D)}\left(p_{1}+\ldots\right)$ corresponding to the overall momentum conservation, and $s \equiv\left(p_{1}+p_{2}\right)^{2}$. The first term on the right-hand side of eqn. (A.1b) denotes the one-loop correlation function in the MS scheme. The above results are invariant under the shifts (2.10), (2.12) as should be.

\section{Appendix B. Equations of motion}

Equations of motion in the context of low energy effective theories has been discussed recently in ref. [6]. We will sketch a derivation of the equations of motion which are necessary in the main text.

Let $T^{I}(\Phi(x))$, a local function of the field $\Phi^{I}$ and its derivatives, be a vector of $\mathrm{O}(\mathrm{N})$. For example, we can take

$$
\begin{aligned}
T^{I}(\Phi)= & a_{0} \Phi^{I}+a_{1,1}\left(\partial_{\mu} \Phi^{J} \partial_{\mu} \Phi^{J}\right) \Phi^{I}+a_{1,2} \partial^{2} \Phi^{I} \\
& + \text { terms with } 4 \text { or more derivatives } .
\end{aligned}
$$


We consider an infinitesimal change of variable

$$
\Phi^{I} \rightarrow \Phi^{\prime I}=\Phi^{I}+\epsilon T^{I}(\Phi)
$$

Since $\Phi^{\prime I}$ must be a unit vector, we must find

$$
\Phi^{I} T^{I}=0
$$

The most general solution to this constraint is given by

$$
T^{I}(\Phi)=\left(\delta^{I J}-\Phi^{I} \Phi^{J}\right) \tilde{T}^{J}(\Phi)
$$

where $\tilde{T}^{I}$ is an arbitrary vector. For example, we have

$$
T^{I}(\Phi)=a\left(\partial^{2} \Phi^{I}+\left(\partial_{\mu} \Phi^{J} \partial_{\mu} \Phi^{J}\right) \Phi^{I}\right)+4 \text { or more derivatives }
$$

Let $S[\Phi]$ be an $\mathrm{O}(\mathrm{N})$ invariant action. For a vector $T^{I}(\Phi)$ which satisfies the condition (B.3), we find

$$
S[\Phi+\epsilon T]-S[\Phi]=\left.\int d^{D} x \epsilon(x) T^{I}(\Phi(x)) \frac{\delta S}{\delta \Phi^{I}(x)}\right|_{n . c .}
$$

where we take $\epsilon(x)$ to be an arbitrary infinitesimal function, and n.c. stands for no constraint, i.e., we do not take into account that $\Phi^{I}$ is a unit vector when we take the derivative. For example, for

$$
S=\int d^{D} x \frac{1}{2 g_{-2}} \partial_{\mu} \Phi^{I} \partial_{\mu} \Phi^{I}
$$

we obtain

$$
\left.\frac{\delta S}{\delta \Phi^{I}(x)}\right|_{n . c}=-\frac{1}{g_{-2}} \partial^{2} \Phi^{I}
$$

Now we consider the correlation function

$$
\left\langle T_{1}^{I_{1}}\left(\Phi\left(x_{1}\right)\right) \ldots T_{n}^{I_{n}}\left(\Phi\left(x_{n}\right)\right)\right\rangle \equiv \int[d \Phi] T_{1}^{I_{1}}\left(\Phi\left(x_{1}\right)\right) \ldots T_{n}^{I_{n}}\left(\Phi\left(x_{n}\right)\right) \exp [-S[\Phi]]
$$

where $T_{i}^{I}$ are arbitrary $\mathrm{O}(\mathrm{N})$ vectors, not necessarily satisfying the constraint (B.3). If we use the dimension regularization, the jacobian of the infinitesimal field transformation (B.2) is unity. Hence, we obtain the following equation of motion:

$$
\begin{aligned}
& \int d^{D} x \epsilon(x)\left\langle\left. T^{I}(\Phi(x)) \frac{\delta S}{\delta \Phi^{I}(x)}\right|_{n . c .} T_{1}^{I_{1}}\left(\Phi\left(x_{1}\right)\right) \ldots T_{n}^{I_{n}}\left(\Phi\left(x_{n}\right)\right)\right\rangle \\
& =\sum_{k=1}^{n}\left\langle T_{1}^{I_{1}}\left(\Phi\left(x_{1}\right)\right) \ldots\left\{T_{k}^{I_{k}}\left(\Phi+\epsilon T\left(\Phi\left(x_{k}\right)\right)\right)-T_{k}^{I_{k}}\left(\Phi\left(x_{k}\right)\right)\right\} \ldots T_{n}^{I_{n}}\left(\Phi\left(x_{n}\right)\right)\right\rangle .
\end{aligned}
$$


For our purposes, it suffices to take the simple action (B.7) and consider two special cases:

(i) $T_{i}^{I}(\Phi)=\Phi^{I}$ and $T^{I}(\Phi)=\partial^{2} \Phi^{I}+\left(\partial \Phi^{J} \partial \Phi^{J}\right) \Phi^{I}$ :

$$
\begin{aligned}
& \int d^{D} x\left\langle\left\{-\partial^{2} \Phi^{I} \partial^{2} \Phi^{I}+\left(\partial \Phi^{J} \partial \Phi^{J}\right)^{2}\right\}(x) \Phi^{I_{1}}\left(x_{1}\right) \ldots \Phi^{I_{n}}\left(x_{n}\right)\right\rangle \\
& =\sum_{k=1}^{n}\left\langle\Phi^{I_{1}}\left(x_{0}\right) \ldots g_{-2}\left\{\partial^{2} \Phi^{I_{k}}+\left(\partial \Phi^{J} \partial \Phi^{J}\right) \Phi^{I_{k}}\right\}\left(x_{k}\right) \ldots \Phi^{I_{n}}\left(x_{n}\right)\right\rangle
\end{aligned}
$$

This gives eqn. (2.11).

(ii) $T_{i}^{I}(\Phi)=\Phi^{I}$ and $T^{I}(\Phi)=\delta^{K I}-\Phi^{K} \Phi^{I}$ (this is a vector for a fixed $K$ ):

$$
\begin{aligned}
& \left\langle\left\{\partial^{2} \Phi^{K}+\left(\partial \Phi^{J} \partial \Phi^{J}\right) \Phi^{K}\right\}(x) \Phi^{I_{1}}\left(x_{1}\right) \ldots \Phi^{I_{n}}\left(x_{n}\right)\right\rangle \\
& \quad=\sum_{k=1}^{n} \delta^{(D)}\left(x-x_{k}\right)\left\langle\Phi^{I_{1}}\left(x_{1}\right) \ldots g_{-2}\left(-\delta^{K I_{k}}+\Phi^{K} \Phi^{I_{k}}\left(x_{k}\right)\right) \ldots \Phi^{I_{n}}\left(x_{n}\right)\right\rangle
\end{aligned}
$$

This gives eqn. (2.9). 


\section{References}

[1] S. Weinberg, Phys. Rev. 166(1968)1568; Physica 96A(1979)327

[2] J. Gasser and H. Leutwyler, Annl. Phys. 158(1984)142

[3] J. Collins, Renormalization (Cambridge University Press, 1984)

[4] T. Appelquist and C. Bernard, Phys. Rev. D23(1981)425

[5] R. Akhoury and Y.-P. Yao, Phys. Rev. D25(1982)3361

[6] C. Arzt, Phys. Lett. B342(1995)189 